Check for updates

Cite this: Phys. Chem. Chem. Phys., 2020, 22, 6449

Received 28th January 2020

Accepted 25th February 2020

DOI: $10.1039 / \mathrm{d} 0 \mathrm{cp} 00465 \mathrm{k}$

rsc.li/pccp

\title{
Structuring of colloidal silica nanoparticle suspensions near water-silica interfaces probed by specular neutron reflectivity
}

\author{
Plinio Maroni, ${ }^{a}$ Manuchar Gvaramia, ${ }^{a}$ Dominik Kosior, (D) ${ }^{a}$ Katarzyna Kubiak, ${ }^{a}$

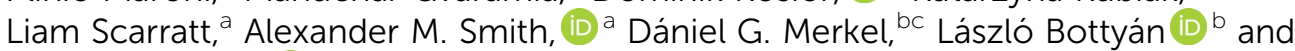 \\ Michal Borkovec (D) *a
}

\begin{abstract}
Structuring of aqueous suspensions of colloidal silica nanoparticles near an isolated planar silica-water interface is studied by specular neutron reflectivity. The reflectivity data clearly show that the suspensions develop a damped, oscillatory concentration profile in the normal direction to the interface. The wavelengths of these oscillations agree well with those independently determined by direct force measurements in the slit-geometry. The reflectivity data further demonstrate that the oscillatory structure persists over several layers and that the first particle layer is separated from the interface by a particle-free region.
\end{abstract}

\section{Introduction}

Interactions between colloidal particles and water-solid interfaces are relevant in a broad range of applications, particularly, in material science, food processing, medicine, or environmental engineering. ${ }^{1-6}$ Several aspects in these processes remain poorly understood, and therefore this topic continues to represent an active research field. A well investigated situation concerns the interaction between charged particles and an oppositely charged interface. ${ }^{5,6}$ In this case, particles deposit rapidly to the substrate due to attractive double layer forces, and form irreversibly bound saturated monolayers with a liquid-like structure. ${ }^{7}$ These lateral structures were analyzed in terms of the random sequential adsorption (RSA) model in detail. ${ }^{3,5}$ The situation, when the particles are like-charged as the substrate, is less well investigated. At lower salt concentrations, particles will not deposit to the surface due to repulsive double layer forces, but deposition may occur at higher salt levels. ${ }^{8,9}$ In the latter situation, thick and porous layers may form due to ripening.

Direct force measurements have recently revealed that concentrated colloidal particle suspensions self-organize when sandwiched between like-charged interfaces, thereby forming layered structures with a characteristic oscillatory profile that

\footnotetext{
${ }^{a}$ Department of Inorganic and Analytical Chemistry, University of Geneva, Sciences II, 30 Quai Ernest-Ansermet, 1205 Geneva, Switzerland. E-mail: michal.borkovec@unige.ch

${ }^{b}$ Wigner Research Centre for Physics, Konkoly-Thege Miklós út 29-33, 1121 Budapest, Hungary

${ }^{c}$ Centre for Energy Research, Konkoly-Thege Miklós út 29-33, 1121 Budapest, Hungary
}

develops normal to the interface. ${ }^{10,11}$ The wavelength $\lambda$ characterizing the spacing within this profile scales as

$$
\lambda \propto \phi_{\mathrm{b}}{ }^{-1 / 3}
$$

where $\phi_{\mathrm{b}}$ is the volume fraction of the particles in the bulk suspension. This dependence occurs since the repulsive double layers around the colloidal particles form a close-packed liquidlike structure. Similar type of ordering was also observed by small angle X-ray scattering in narrow slits for hard spheres and charged particles. ${ }^{12,13}$ This structuring has been independently confirmed in the bulk phase by scattering experiments through the appearance of a structural peak..$^{10,11,14,15}$

Numerous theoretical studies suggest that similar layered structures should also form near isolated interfaces in hard-sphere or charged colloidal suspensions. ${ }^{16-19}$ However, the experimental evidence is scarce. We are only aware of two X-ray reflectivity studies addressing this issue. ${ }^{20,21}$ While these studies indeed suggest the formation of layered structures, variations in the particle concentration were not reported, and for the isolated interface the important question concerning the concentration dependence of the wavelength as suggested by eqn (1) remains open.

The present study addresses this question by means of neutron reflectivity. While neutron reflectivity is an established tool to study adsorption of polymers and surfactants to watersolid interfaces, ${ }^{22-27}$ we are only aware of three neutron reflectivity studies of similar surface layering, albeit in completely different systems, namely in solutions of block copolymer micelles ${ }^{28,29}$ and of proteins. ${ }^{30}$ Here, we exploit the potential of neutron reflectivity to probe layered structures in aqueous suspensions of negatively charged silica nanoparticles near a like-charged water-silica interface. This technique is indeed well suited to study this system, and 
reveals the formation of layered structures near this interface. Quantitative analysis of such reflectivity curves permits to estimate the actual concentration profile with good confidence. In particular, we find that the wavelength extracted from reflectivity profiles indeed follows the dependence suggested by eqn (1).

\section{Experimental}

\section{Materials}

Silica particles were purchased as an aqueous suspension (Ludox HS40, Sigma-Aldrich). The mass fraction of the particles was determined by drying overnight at $110{ }^{\circ} \mathrm{C}$ to constant weight, whereby the value provided by the manufacturer of 0.40 could be confirmed. The particle size distribution was determined with dried suspensions with transmission electron microscopy (TEM, Tecnai G2, FEI) and atomic force microscopy (AFM, MFP-3D, Asylum Research). Both methods give consistently an average diameter of $130 \pm 2 \AA$ and a polydispersity expressed as the coefficient of variation of $0.17 \pm 0.03$. The density of the silica suspension was measured by weighing an object of known mass and volume in the suspension (Easy Dyne K20, Krüss), and the density of the particles was extracted to be $2.29 \mathrm{~g} \mathrm{~mL}^{-1}$ by assuming ideal mixing. The particle suspensions were further analyzed at a concentration of $10 \mathrm{mg} \mathrm{L}^{-1}$ in $1.0 \mathrm{mM} \mathrm{NaCl}$ solution by dynamic and electrophoretic light scattering (Zetasizer Nano ZS, Malvern). From the second cumulant method and the Stokes-Einstein relation one finds a hydrodynamic diameter of $163 \pm 7 \AA$, and by invoking the Henry model an electrokinetic potential of $-69 \mathrm{mV}$. When the hydrodynamic diameter is calculated from ratio of the sixth and fifth moments of the size distribution obtained by microscopy, ${ }^{31}$ one finds a hydrodynamic diameter of $138 \AA$, which is reasonable agreement with the value measured by dynamic light scattering reported above. A batch of silica particles, which was also labeled as HS40, was characterized earlier with TEM by Zeng et $a .^{32}$ and Ludwig et al. ${ }^{33}$ These authors systematically find somewhat larger particle size and lower densities, from which we conclude that the particle batch used here is similar, but not identical, to the one described by these authors.

Prior to use, the silica suspensions were purified by ultrafiltration in a stirred cell (Amicon, Millipore) with ultragel membranes with a molecular mass cut-off of $5 \mathrm{~kg} \mathrm{~mol}^{-1}$ (Amicon, Millipore) against pure water. The process was terminated when the conductivity of the filtrate dropped below $20 \mu \mathrm{S} \mathrm{cm}^{-1}$, which was usually the case after one week. The particle concentrations in the dialyzed suspensions were also determined by drying, as described above. Particle suspensions of lower particle concentration were prepared by mixing dialyzed stock suspension and water by weight. The particle volume fraction was calculated by assuming ideal mixing.

Neutron reflectivity was carried out with two similar polished silicon blocks (hereinafter denoted as block A and B) of dimensions $85 \mathrm{~mm} \times 50 \mathrm{~mm} \times 20 \mathrm{~mm}$ each (Andrea Holm GmbH, Tann). The thickness of terminating silica layer was measured for both blocks by nulling ellipsometry (Multiskop, Optrel) in air and was found to be $23 \pm 1 \AA$. The surface roughness was determined by topographic imaging with the AFM (MFP-3D, Asylum Research), and the root mean square (RMS) roughness was determined as 5.0 A. Purified water (MilliQ, Millipore) was used throughout. All experiments were carried out at room temperature $20 \pm 2{ }^{\circ} \mathrm{C}$.

\section{Neutron reflectivity}

The measurements were carried out on the GINA reflectometer equipped with a solid-liquid cell at the Budapest Neutron Center, Hungary. A scheme of the experiment is shown in Fig. 1. The unpolarized monochromatic neutron beam with a wavelength $\lambda_{0}=4.61 \AA$ was obtained using a pyrolitic graphite monochromator and a cryo-cooled beryllium filter. The beam was further constrained by two cadmium slits positioned $0.30 \mathrm{~m}$ and $1.30 \mathrm{~m}$ upstream from the sample (slit 1 and 2). The beam is impinging the vertically positioned solid-water interface at an angle of incidence $\theta$ through the Si-block. The specularly reflected beam is selected by an other slit (slit 3), passes an evacuated flight tube, and is analyzed with a position sensitive detector centered at an angle of $2 \theta$. More details about the instrument can be found elsewhere. ${ }^{34,35}$

Prior to each experiment, the silicon block was cleaned by sonication in ethanol for $20 \mathrm{~min}$, and then followed by an oxygen treatment in a Pico plasma system (Diener Electronic $\mathrm{GmbH}$, Ebhausen) at a power of $200 \mathrm{~W}$ and a pressure of $0.2 \mathrm{mbar}$ for $10 \mathrm{~min}$. Cleaned blocks were stored in evacuated desiccator prior to use. The cleaned silicon block was clamped against a Teflon trough with a volume of about $8 \mathrm{~mL}$ and sandwiched between two aluminum plates in a similar arrangement as described earlier. ${ }^{36}$ The trough was then filled with the suspension in question through an inlet port avoiding the formation of air bubbles. The Si-block was aligned as follows. The zero angle and the region of interest (ROI) were set on the $2 \mathrm{D}$ detector with the primary beam in absence of the sample. The cell was then mounted on the $\theta$-goniometer. The detector angle was set to center the ROI to $0.4^{\circ}$. The incident angle and the transversal position of the Si surface were adjusted relative to the primary beam until the specularly reflected beam got centered in the ROI. The actual incident angle was then redefined to half of the detector angle.

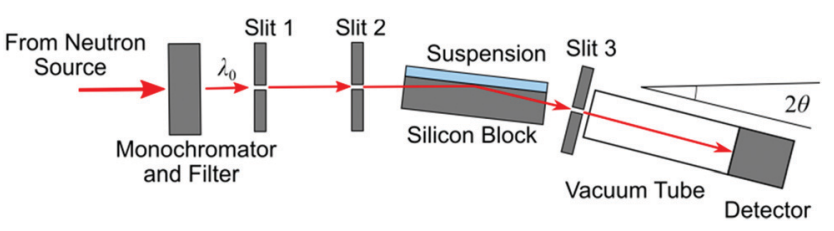

Fig. 1 Schematic top-view of the GINA neutron reflectometer setup. The neutron beam is made monochromatic with a pyrolitic graphite monochromator and a beryllium filter and passes through slits 1 and 2 . Then it impinges the solid-water interface oriented at an incident angle $\theta$. The specularly reflected beam is selected with the slit 3, and is analyzed with a position sensitive detector centered at an angle $2 \theta$. Further details concerning the instrument are given elsewhere. ${ }^{34,35}$ 
The intensity of specularly reflected neutrons was measured as a function of the magnitude of the scattering vector $q$, which was calculated as $^{24}$

$$
q=\frac{4 \pi}{\lambda_{0}} \sin \theta
$$

Reflectivity was typically recorded during 8-12 hours for angles of incidence $0.15-3.3^{\circ}$, which leads to a $q$-range of $0.007-0.180 \AA^{-1}$. At low angles, only a part of the primary beam hits the sample. The full beam is only incident at angles above $\theta_{\mathrm{m}}=\arctan (h / L)$, where $h=0.6 \mathrm{~mm}$ is the slit width and $L=75 \mathrm{~mm}$ is the length of the illuminated part of the sample. For angles $\theta<\theta_{\mathrm{m}}$ the measured intensities are corrected by a factor of $\sin \theta / \sin \theta_{\mathrm{m}}$ for the beam footprint.

The spread $\Delta q$ in the scattering vector was estimated from the relative contributions from the wavelength and the angle of incidence, namely ${ }^{37}$

$$
\left(\frac{\Delta q}{q}\right)^{2}=\left(\frac{\Delta \lambda_{0}}{\lambda_{0}}\right)^{2}+\left(\frac{\Delta \theta}{\theta}\right)^{2}
$$

where $\Delta \lambda_{0} / \lambda_{0}$ is the relative spread in the wavelength of about $\Delta \lambda_{0} / \lambda_{0}=0.01$, and $\Delta \theta=\arctan (h / D)$ where $D=1000 \mathrm{~mm}$ is the distance between the slits. Depending on the angle of incidence, we thus have a relative spread $\Delta q / q$ ranging from $0.01-0.20$, whereby the smallest spread is found for the largest angles. ${ }^{35}$

\section{Direct force measurements}

Forces involving colloidal silica micro-particles were measured with a closed-loop AFM (MFP-3D, Asylum Research, USA) mounted on an inverted optical microscope (Olympus IX 73). The silica micro-particles (Bangs Laboratories Inc., USA) with a diameter of $5.2 \mu \mathrm{m}$ were attached to tipless cantilevers with epoxy glue in air. Particles were also sprinkled over cleaned quartz substrates and then heat-treated together with the cantilevers at $1150{ }^{\circ} \mathrm{C}$ for 3 hours. This sintering attaches the particles, but also leads to minor shrinkage to a diameter of $4.4 \mu \mathrm{m}$, reduces their surface roughness, and removes the remaining glue. ${ }^{38}$ The RMS roughness of similarly prepared silica particles was $0.7 \mathrm{~nm}$ as described previously. ${ }^{39}$ Immediately prior to force measurements, silica substrates and cantilevers were thoroughly rinsed with ethanol, subsequently with water, then dried, and cleaned in air plasma for 20 minutes. To carry out force measurements in the sphere-sphere geometry, a pair of particles was centered with the optical microscope. Subsequent approach-retraction cycles were then measured at a velocity of $500 \mathrm{~nm} \mathrm{~s}^{-1}$. The deflection signal was converted to force profiles by subtracting the baseline and the constant compliance region, and by considering the respective spring constants of $0.2-0.5 \mathrm{~N} \mathrm{~m}^{-1}$. The spring constant was determined from the lateral dimensions of the cantilever and its frequency response as described by Sader et al. ${ }^{40}$ Forces obtained from about 100 repeated approach and retraction curves were averaged, leading to a force resolution of approximately $2 \mathrm{pN}$. The resulting force curves were block averaged further. The sphere-sphere geometry was used to measure forces in silica suspensions. The same geometry was used to determine the diffuse layer potential for the micro-particle in $1.0 \mathrm{mM} \mathrm{NaCl}$ solutions with $\mathrm{pH}$ near 6 . In the latter case, the force profile was interpreted within the PoissonBoltzmann (PB) model of the electrical double layer. ${ }^{41,42}$ The forces between the bare silica micro-particles were fitted in the symmetric geometry, and one obtains a diffuse layer potential of $-65 \mathrm{mV}$. The calibrated probe particle was then used to measure the diffuse layer potential of the silicon block in sphere-plane geometry. The respective force curve was fitted with the PB model in the asymmetric geometry, whereby the diffuse layer potential of the probe particle was held fixed. This procedure yields a diffuse layer potential of the silicon block of $-42 \mathrm{mV}$. These measurements thus demonstrate that the silicon block is also negatively charged. More details on similar force measurements can be found elsewhere. ${ }^{39,42}$

\section{Results and discussion}

The present study reports specular neutron reflectivity measurements in aqueous suspensions of silica nanoparticles near a water-silica interface. This interface was realized with a polished silicon ( $\mathrm{Si})$ block, which spontaneously forms a thin silica $\left(\mathrm{SiO}_{2}\right)$ layer on its surface. In pure water, this silica surface acquires negative charge. Neutron reflectivity shows a clear signature of an oscillatory concentration profile of the nanoparticles near the interface. Their wavelength is in good agreement with measurements of the oscillatory forces acting between two silica surfaces in the same suspensions.

\section{Neutron reflectivity}

Measured reflectivity from the bare surface in pure water is shown in Fig. 2a. In this case, the reflectivity approximately decreases as $q^{-4}$ as expected for a sharp interface. The profile recorded in an aqueous suspension of the silica particles with a bulk volume fraction of 0.10 is shown in Fig. $2 \mathrm{~b}$. The reflectivity curve now features a characteristic shoulder near $0.025 \AA^{-1}$. This shoulder is a signature of a decaying oscillatory structure near the interface, and the corresponding wavelength $\lambda$ can be estimated from the Bragg condition as $\lambda=2 \pi / q \approx 250 \AA$ A. Two different silicon blocks $\mathrm{A}$ and $\mathrm{B}$ have been used in the experiments, and as shown in Fig. 2, they both yield identical results within experimental error. The reflectivity was also measured after rinsing the cell, which was previously filled with the particle suspension, with pure water. The observed reflectivity curve was indistinguishable from the one of the bare substrate shown in Fig. 2a.

The experimental reflectivity data can be interpreted quantitatively by means of the Abele's matrix method, whereby we use the algorithm implemented in Motofit. ${ }^{43}$ The scattering intensity was corrected for the beam footprint and converted to the reflectivity by fitting an overall scale factor and by including a common background. Smearing of the wave vector is included in the reflectivity calculation as shown in eqn (3). The bare interface could be well fitted with a silica slab that is sandwiched between silicon and water, see Fig. 2a. One extracts the thickness of $24 \pm 2 \AA$, which is in good agreement with the 

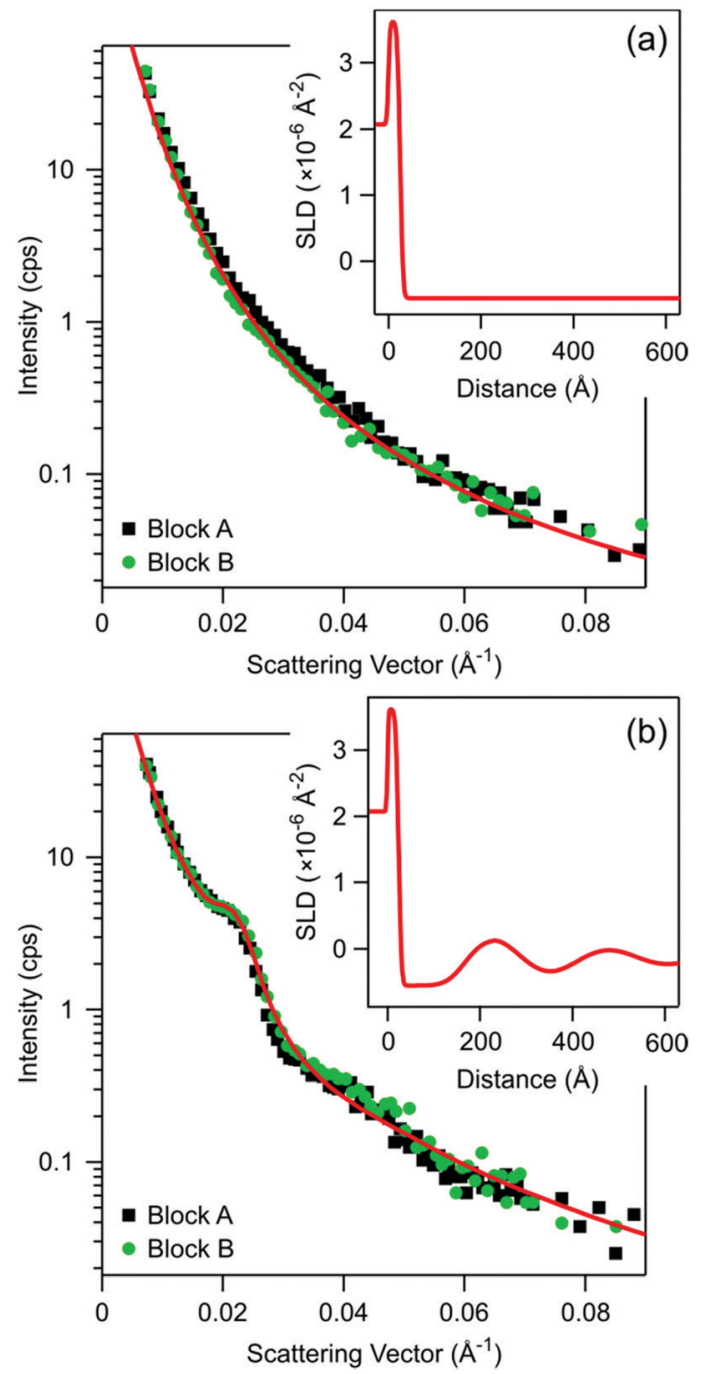

Fig. 2 Reflected intensity expressed in counts of neutrons per second (cps) versus the magnitude of the scattering vector $q$ in aqueous suspensions for two different silicon blocks A and B. Solid lines are best fits as described in the text. The extracted scattering length density (SLD) profiles are shown as insets whereby the distance origin is chosen at the silicasilicon interface. (a) Pure water and (b) aqueous particle suspension of a bulk volume fraction $\phi_{\mathrm{b}}=0.10$.

value obtained by ellipsometry. The roughness of the silicasilicon and water-silica interfaces is modeled in a standard way by means of an error function ${ }^{43}$ with an RMS roughness of $2 \AA$ and $5 \AA$, respectively, whereby the latter has been measured with the AFM. The scattering length density (SLD) for silicon, silica, and water of $2.07 \times 10^{-6} \AA^{-2}, 3.62 \times 10^{-6} \AA^{-2}$, and $-5.59 \times 10^{-7} \AA^{-2}$ were calculated from the tabulated scattering lengths, atomic masses, and corresponding densities. ${ }^{44,45}$ The corresponding SLD profile is shown as inset in Fig. 2a.

To model the structuring of the particle suspension near the interface, we have included into the fitting procedure the following damped oscillatory profile of the particle volume fraction

$$
\phi(x)=\Delta \phi \mathrm{e}^{-x / \xi} \cos (2 \pi x / \lambda)+\phi_{\mathrm{b}}
$$

where $\Delta \phi$ is the difference between the maximal and bulk volume fraction, $\xi$ the correlation length, and $x$ is the distance from the interface, whereby its origin is taken at the first peak of the profile. Such exponentially damped oscillatory profile is expected due to the appearance of complex poles determining the decay of correlations in hard-sphere and charged particle fluids. ${ }^{16-18}$ To obtain a quantitative fit of the neutron data, it was necessary to introduce a particle-free layer containing pure water of thickness $d$ next to the water-silica interface. Such a particle-free layer should occur due to electrostatic repulsion between the charged particles and the like-charged interface and its existence has further been suggested on theoretical grounds. ${ }^{19} \mathrm{~A}$ smooth transition between this layer and the profile given in eqn (4) was obtained empirically by means of an error function with an apparent RMS roughness of $\lambda / 8$, which was centered at $x=-\lambda / 4$. The oscillatory profile given in eqn (4) is then invoked for $x>0$. The SLD of the suspension was calculated from the volume fraction profile and the respective SLDs of water and silica given above by assuming ideal mixing.

The parameters were extracted from the least squares-fit of the experimental data, which is shown in Fig. $2 \mathrm{~b}$. The resulting values are a wavelength of $\lambda=252 \AA$, a correlation length of $\xi=383 \AA$, an amplitude of the oscillatory volume fraction $\Delta \phi=0.062$, and the thickness of the particle free layer of $d=142 \AA$ A. Note that the fitted wavelength is in good agreement with the above estimate based on the position of the shoulder from the Bragg's condition. The calculate SLD profile is again shown as inset in Fig. 2 b. The extracted volume fraction profile is further plotted in Fig. 3. This profile resembles the one used to analyze X-ray reflectivity data in a similar system, ${ }^{20}$ but contains a much smaller number of adjustable parameters, and encompasses the expected asymptotic decay of the concentration profile. We have further attempted to improve the fits with eqn (4) by introducing a phase shift within the argument of the cosine function, but the best fit resulted when this shift was set to zero as given.

Fig. 4 shows additional reflectivity curves at different particle concentrations, whereby the profiles are displaced for clarity. With increasing concentration, the shoulder in the profiles becomes increasingly pronounced, and at a bulk volume fraction of 0.16 a clear structural peak becomes evident. At a volume fraction of 0.20 , the structural peak becomes very pronounced, and it is accompanied by a second peak at higher $q$-values. At these concentrations, the sample probably starts to crystallize. Colloidal crystallites were also visually apparent in the optical microscope. Formation of crystals might be promoted by the presence of the interface, and induce the Bragg reflections observed. Except for this highest concentration, the data can be again well fitted with the same model as discussed above.

As evident from the shift of the shoulder in the reflectivity spectrum, the oscillation wavelength decreases with increasing concentration, as shown in Fig. 5a. Indeed, the wavelength drops in a monotonic fashion from about $340 \AA$ at a bulk volume fraction of about 0.05 to about $220 \AA$ at 0.16 . As discussed in the 

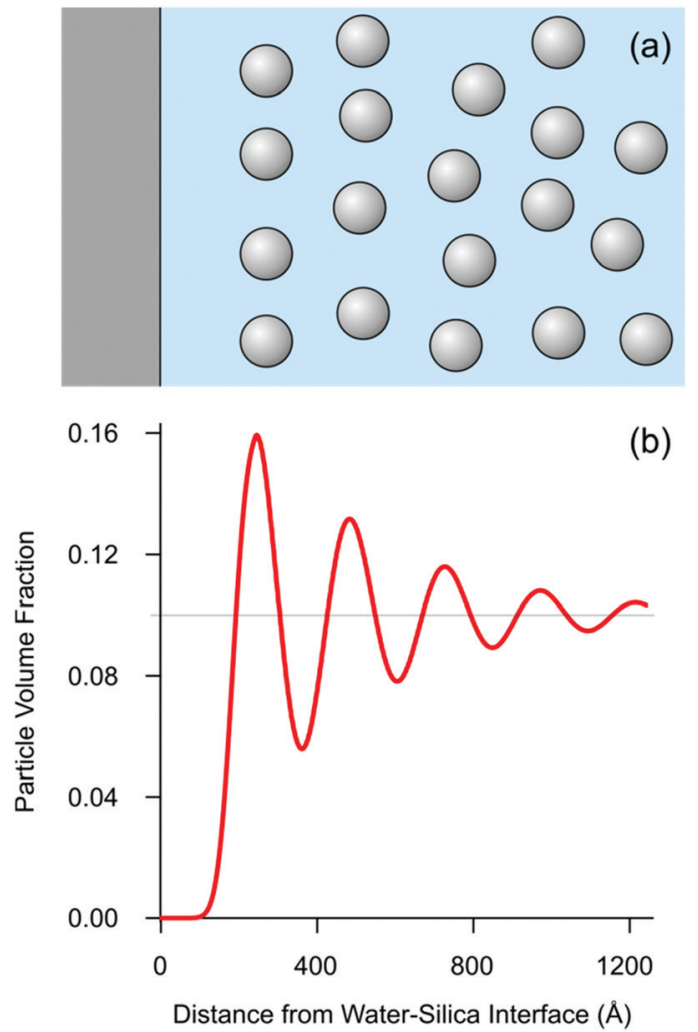

Fig. 3 Self-organization of the silica nanoparticle suspension in the normal direction of the silica-water interface. (a) Schematic representation of the suspension next to the interface. (b) Calculated volume fraction profile normal to the interface for the conditions given in Fig. 1b.

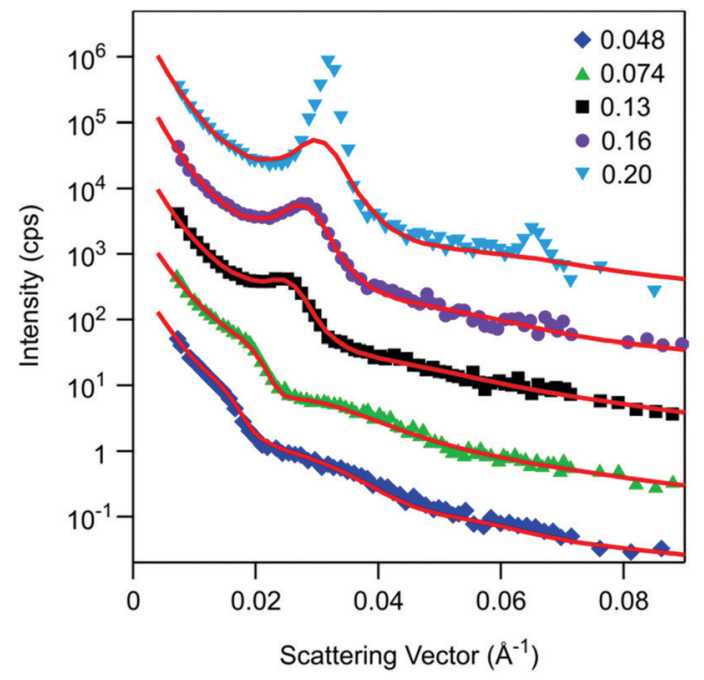

Fig. 4 Reflected intensity expressed in counts of neutrons per second (cps) versus the magnitude of the scattering vector $q$ in aqueous suspensions at different bulk volume fractions indicated. The profile corresponding to the lowest volume fraction is plotted on the actual scale, while for each higher concentration the profiles are always multiplied by a factor of 10. Solid lines are best fits as described in the text.

introduction, the measured wavelength scales as suggested by eqn (1). This relation is also shown in Fig. 5a, whereby it appears as a straight line in the doubly-logarithmic plot with a slope of $-1 / 3$. The measured wavelength typically exceeds the particle diameter by a factor of $2-3$, which suggests the importance of electrostatic repulsion in the self-organization of the nanoparticles near the water-solid interface.

The correlation length fluctuates randomly throughout the entire concentration series with a mean and a standard deviation of $\xi=304 \pm 60 \AA$. The data can be well interpreted by fixing this value to the mean. While this value remains roughly constant, the wavelength decreases with increasing particle concentration. As a consequence, the ratio $\xi / \lambda$ thus increases from about 0.9 to 1.4 , which indicates a progressive protrusion of the oscillatory structure into the bulk.

The thickness of the particle-free layer is shown in Fig. 5b, and it decreases with increasing particle concentration. This thickness is roughly corresponds to $\lambda / 2$. However, the decrease
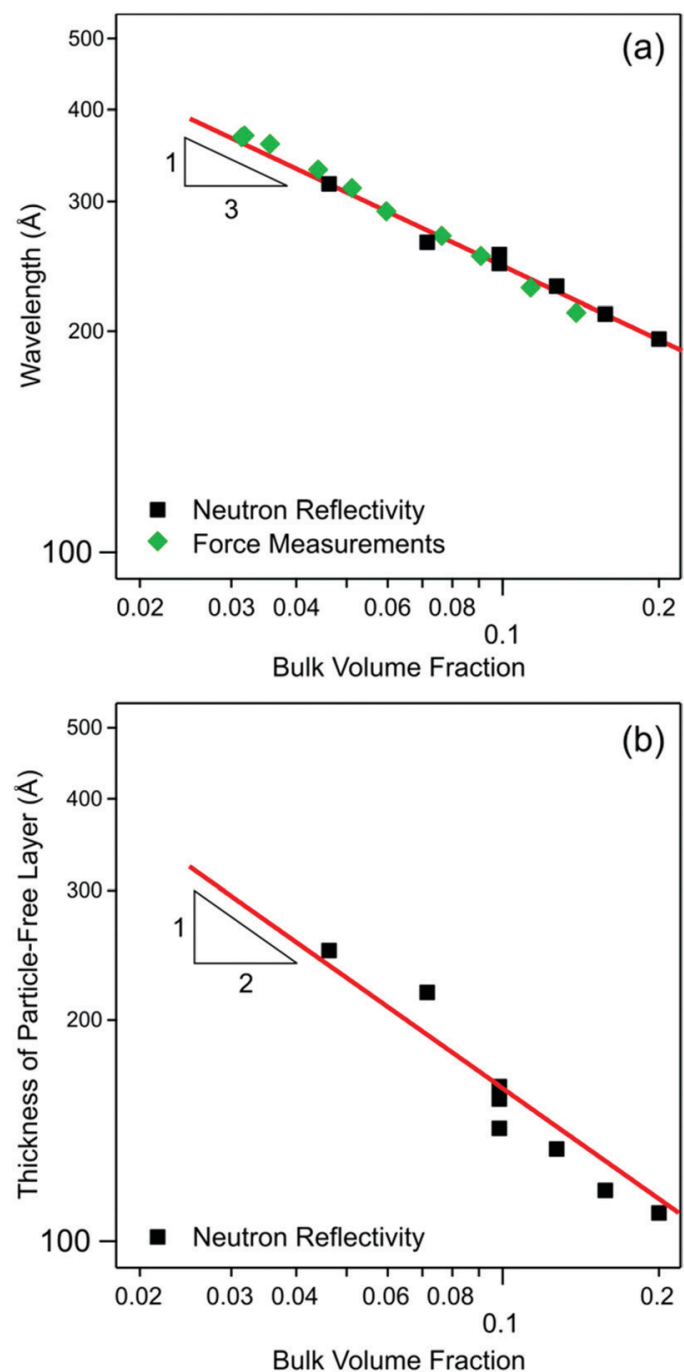

Fig. 5 Doubly logarithmic plots of various parameters extracted from neutron reflectivity versus bulk volume fraction $\phi_{\mathrm{b}}$. (a) Wavelength $\lambda$ together with data from direct force measurements and best fit according to eqn (1). (b) Thickness of the particle free layer $d$ together with the scaling law given in eqn (5). 
is more pronounced than what might be expected based on eqn (1), but one rather finds a scaling law

$$
d \propto \phi_{\mathrm{b}}{ }^{-1 / 2}
$$

This dependence is also shown in Fig. 5b. We suspect that this dependence originates from the electrostatic repulsion of the nanoparticles from the like-charged interface. The decrease of its thickness can be then interpreted through the increasing concentration of the counter-ions and thus an increase in electrostatic screening. The Debye length, which characterizes the thickness of the electrical double layer, increases with the inverse of the square root of the ionic concentrations, and thus this relation might rationalize the exponent of $-1 / 2 .{ }^{41}$ Our observation of the particle-free layer is in line with recent quartz crystal microbalance (QCM) measurements of suspensions of negatively charged polystyrene nanoparticles. ${ }^{46}$ That study concluded that near a water-silica interface a particle-free layer with a thickness of few particle diameters is present. Such particle-free layers were also reported in theoretical studies of charged particle suspensions in contact with a like-charged wall. $^{19}$

We further note that the difference between the maximal and bulk volume $\Delta \phi$ increases with increasing particle concentration, and is comparable to the bulk volume fraction at the highest concentration investigated.

\section{Direct force measurements}

An independent confirmation of the proposed oscillatory ordering can be made with direct force measurements. Forces acting across the same silica suspensions sandwiched between surfaces of two silica micro-particles were measured with the AFM in the sphere-sphere geometry. Fig. 6 shows the measured forces $F$ are shown versus the displacement distance $D$, whereby the offset was chosen such that the piezo displacement $D$ is zero at the first maximum in the force profile. Their oscillatory profile reveals a similar type of layering as suggested by the neutron reflectivity experiments, and reflects the layering of the colloidal particles sandwiched in between the two interfaces. We further note that the approach and retraction force profiles are identical within experimental error, which confirms that this force originates from the structuring of the particle suspension, and that these structures relax rapidly on the time scale of the force experiment.

The forces clearly show a damped oscillatory structure, and at larger displacements they can be well fitted with the relation

$$
F(D)=A \mathrm{e}^{-D / \xi} \cos (2 \pi D / \lambda)
$$

where $A$ is the amplitude of the force. Representative fits are also shown in Fig. 6. The dependence suggested by eqn (6) is well obeyed at larger distances, but discrepancies are observed at smaller separations, especially at higher concentrations.

Fig. 5a compares the wavelengths obtained from the direct force measurements with the AFM with the ones extracted from the neutron reflectivity. Within experimental noise, the values obtained with these two entirely independent techniques are in excellent agreement. The correlation length extracted from the
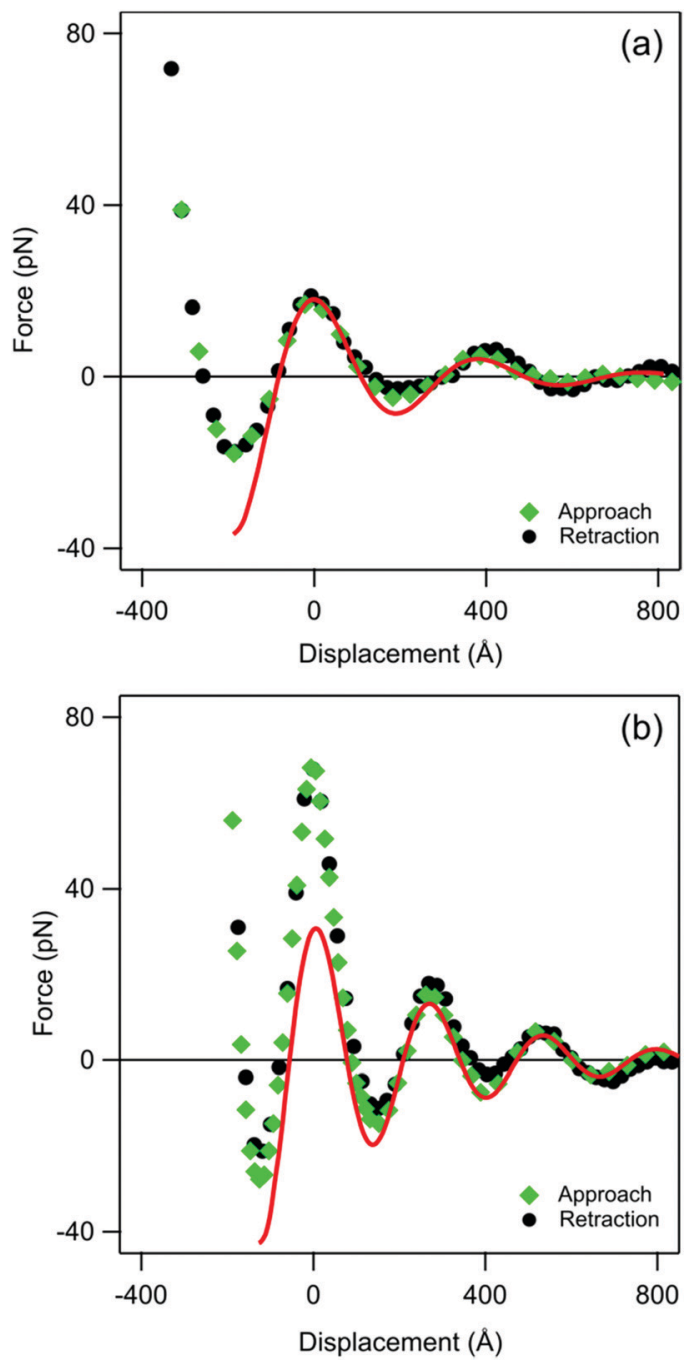

Fig. 6 Oscillatory forces versus piezo displacement between two silica microparticles measured at different nanoparticle concentrations with the colloidal probe technique upon approach and retraction. The offset in the displacement is defined to be zero at the first maximum of the force. The solid line is best fit with eqn (6) starting with the second maximum. The wavelength extracted from such profiles is shown in Fig. 4a. Volume fraction of (a) 0.025 and (b) 0.076 .

force curves shows no clear trends with the concentration, and scatters around a mean value with a standard deviation of $\xi=250 \pm 50 \AA$. This number is somewhat smaller, but within the error bar of the one found by neutron reflectivity of $\xi=304 \pm 60 \AA$, see above. The amplitude of the force increases with increasing particle concentration. This increase is in qualitative agreement with the observed trend in the peak volume fraction. Note that the use of the piezo displacement instead of the absolute separation distance does not affect the determination of the parameters $\lambda$ and $\xi$. This choice was made due to difficulties in locating the precise contact point of the two surfaces in the particle suspensions.

Very similar oscillatory force profiles were reported by von Klitzing and coworkers. ${ }^{10,32,33}$ These authors have measured forces in the sphere-plane geometry in suspensions of similar 
silica nanoparticles. As explained in the experimental part, however, the batch of the particles used was probably different from the present one. Nevertheless, the reported wavelengths and correlation lengths were very similar to the ones reported here. The fact that layering can lead to oscillatory forces has further been established theoretically for hard spheres and charged colloidal particles, ${ }^{19,47}$ as well as experimentally in other systems, such as polyelectrolytes or micelles. ${ }^{4-50}$

The agreement between these parameters obtained from direct force measurements and neutron reflectivity clearly demonstrate the existence of the proposed oscillatory structures, be it between two surfaces or near an isolated one. While the silica surfaces of the microparticles are surely similar to the silica layer of the silicon block used in the neutron experiments, they may not be identical. However, it was shown earlier that the same wavelength dependence was observed when the confinement was achieved with an air-water interface, and provided the interface is negatively charged, the influence of the type of interface is probably minor. ${ }^{11,32,51}$

\section{Conclusions}

Neutron reflectivity provides clear evidence that suspensions of colloidal silica nanoparticles show a damped, oscillatory concentration profile normal to a planar silica-water interface. The wavelength of these oscillations decreases with increasing concentration, and typically exceeds the particle diameter by a factor of 2-3. The measured wavelengths obtained by neutron reflectivity agree very well with the one determined by direct force measurements with the AFM in the slit-geometry, which suggest that in both geometries the self-organization of the nanoparticles is governed by the same principles. The reflectivity further indicates that the oscillatory structure persists through few layers into the bulk and that its onset is separated from the interface by a particle free gap, whose width is about half the wavelength. The present study thus demonstrates that the oscillatory concentration profile for an isolated surface has the same characteristics as for the slit-geometry. Such oscillatory profiles further reflect the liquid-like structuring in the bulk suspension. On the other hand, the particle-free gap seems to be specific to the nature of the surface. We hope that the present experimental results can be soon confronted with detailed theoretical models, and thus provide a better understanding of structuring of colloidal suspensions near interfaces.

\section{Conflicts of interest}

There are no conflicts of interest to declare.

\section{Acknowledgements}

This research was supported by the Swiss National Science Foundation through the project no. 178759, University of Geneva, Microsystems Laboratory of Centre for Energy Research in Budapest, and the Budapest Neutron Centre. We thank Johannes
Bookhold, Magda Erös, Péter Fürjes, Michael Ludwig, Gaetano Mangiapia, Róbert Mészáros, Zsuzsanna Sík, and Regine von Klitzing, for useful suggestions, sample preparation, chemicals, and preliminary measurements.

\section{References}

1 R. Mezzenga, P. Schurtenberger, A. Burbidge and M. Michel, Nat. Mater., 2005, 4, 729-740.

2 K. A. Dunphy Guzman, M. R. Taylor and J. F. Banfield, Environ. Sci. Technol., 2006, 40, 1401-1407.

3 B. Senger, J. C. Voegel and P. Schaaf, Colloids Surf., A, 2000, 165, 255-285.

4 E. Matijevic and S. V. Babu, J. Colloid Interface Sci., 2008, 320, 219-237.

5 Z. Adamczyk, Adv. Colloid Interface Sci., 2003, 100, 267-347.

6 M. Elimelech, J. Gregory, X. Jia and R. A. Williams, Particle Deposition and Aggregation: Measurement, Modeling, and Simulation, Butterworth-Heinemann Ltd, Oxford, 1995.

7 M. Semmler, E. K. Mann, J. Ricka and M. Borkovec, Langmuir, 1998, 14, 5127-5132.

8 M. Elimelech and C. R. O'Melia, Langmuir, 1990, 6, 1153-1163. 9 N. Ryde, N. Kallay and E. Matijevic, J. Chem. Soc., Faraday Trans., 1991, 87, 1377-1381.

10 S. H. L. Klapp, Y. Zeng, D. Qu and R. von Klitzing, Phys. Rev. Lett., 2008, 100, 118303.

11 Y. Zeng and R. von Klitzing, Soft Matter, 2011, 7, 5329-5338.

12 K. Nygard, R. Kjellander, S. Sarman, S. Chodankar, E. Perret, J. Buitenhuis and J. F. van der Veen, Phys. Rev. Lett., 2012, 108, 37802 .

13 D. K. Satapathy, O. Bunk, K. Jefimovs, K. Nygard, H. Guo, A. Diaz, E. Perret, F. Pfeiffer, C. David, G. H. Wegdam and J. F. van der Veen, Phys. Rev. Lett., 2008, 101, 136103.

14 H. Versmold, U. Wittig and W. Hartl, J. Phys. Chem., 1991, 95, 9937-9940.

15 T. Gisler, S. F. Schulz, M. Borkovec, H. Sticher, P. Schurtenberger, B. D’Aguanno and R. Klein, J. Chem. Phys., 1994, 101, 9924-9936.

16 R. Evans, R. J. F. L. de Carvalho, J. R. Henderson and D. C. Hoyle, J. Chem. Phys., 1994, 100, 591-603.

17 C. Grodon, M. Dijkstra, R. Evans and R. Roth, Mol. Phys., 2005, 103, 3009-3023.

18 R. M. Adar, S. A. Safran, H. Diamant and D. Andelman, Phys. Rev. E, 2019, 100, 42615.

19 P. Gonzalez-Mozuelos, J. Alejandre and M. Medina-Noyola, J. Chem. Phys., 1992, 97, 8712-8721.

20 K. Nygard and O. Konovalov, Soft Matter, 2012, 8, 5180-5186. 21 A. Madsen, O. Konovalov, A. Robert and G. Grubel, Phys. Rev. E: Stat., Nonlinear, Soft Matter Phys., 2001, 64, 061406.

22 J. R. Howse, R. Steitz, M. Pannek, P. Simon, D. W. Schubert and G. H. Findenegg, Phys. Chem. Chem. Phys., 2001, 3, 4044-4051.

23 M. Cardenas, H. Wacklin, R. A. Campbell and T. Nylander, Langmuir, 2011, 27, 12506-12514.

24 J. Penfold and R. K. Thomas, Curr. Opin. Colloid Interface Sci., 2014, 19, 198-206. 
25 L. Braun, M. Uhlig, R. von Klitzing and R. A. Campbell, Adv. Colloid Interface Sci., 2017, 247, 130-148.

26 M. Kolasinska, R. Krastev, T. Gutberlet and P. Warszynski, Langmuir, 2009, 25, 1224-1232.

27 R. Steitz and R. Dahint, Adv. Eng. Mater., 2011, 13, 773-783.

28 M. C. Gerstenberg, J. S. Pedersen, J. Majewski and G. S. Smith, Langmuir, 2002, 18, 4933-4943.

29 N. Wolff, S. Gerth, P. Gutfreund and M. Wolff, Soft Matter, 2014, 10, 8420-8426.

30 M. W. A. Skoda, F. Schreiber, R. A. J. Jacobs, J. R. P. Webster, M. Wolff, R. Dahint, D. Schwendel and M. Grunze, Langmuir, 2009, 25, 4056-4064.

31 M. Kobayashi, F. Juillerat, P. Galletto, P. Bowen and M. Borkovec, Langmuir, 2005, 21, 5761-5769.

32 Y. Zeng, S. Grandner, C. L. P. Oliveira, A. F. Thunemann, O. Paris, J. S. Pedersen, S. H. L. Klapp and R. von Klitzing, Soft Matter, 2011, 7, 10899-10909.

33 M. Ludwig, M. U. Witt and R. von Klitzing, Adv. Colloid Interface Sci., 2019, 269, 270-276.

34 L. Bottyan, D. G. Merkel, B. Nagy, J. Fuzi, S. Sajti, L. Deak, G. Endroczi, A. V. Petrenko and J. Major, Rev. Sci. Instrum., 2013, 84, 15112.

35 L. Bottyan, D. G. Merkel, B. Nagy and J. Major, Sci. Rev., 2012, 23, 21-24.

36 G. Fragneto-Cusani, J. Phys.: Condens. Matter, 2001, 13, 4973-4989.

37 N. K. Pleshanov, Nucl. Instrum. Methods Phys. Res., Sect. A, 2016, 820, 146-155.
38 V. Valmacco, M. Elzbieciak-Wodka, C. Besnard, P. Maroni, G. Trefalt and M. Borkovec, Nanoscale Horiz., 2016, 1, 325-330.

39 M. Moazzami-Gudarzi, P. Maroni, M. Borkovec and G. Trefalt, Soft Matter, 2017, 13, 3284-3295.

40 J. E. Sader, J. W. M. Chon and P. Mulvaney, Rev. Sci. Instrum., 1999, 70, 3967-3969.

41 W. B. Russel, D. A. Saville and W. R. Schowalter, Colloidal Dispersions, Cambridge University Press, Cambridge, 1989.

42 F. J. Montes Ruiz-Cabello, G. Trefalt, P. Maroni and M. Borkovec, Phys. Rev. E: Stat., Nonlinear, Soft Matter Phys., 2014, 90, 012301.

43 A. Nelson, J. Appl. Crystallogr., 2006, 39, 273-276.

44 V. F. Sears, Neutron News, 1992, 3, 26-37.

45 R. C. Weast and M. J. Astle, CRC Handbook of Chemistry and Physics, CRC Press, New York, 1980.

46 M. S. Hellsing, A. R. Rennie, M. Rodal and F. Hook, Langmuir, 2019, 35, 222-228.

47 A. Trokhymchuk, D. Henderson, A. Nikolov and D. T. Wasan, Langmuir, 2001, 17, 4940-4947.

48 P. Richetti and P. Kekicheff, Phys. Rev. Lett., 1992, 68, 1951-1954.

49 A. J. Milling and K. Kendall, Langmuir, 2000, 16, 5106-5115.

50 M. Moazzami-Gudarzi, T. Kremer, V. Valmacco, P. Maroni, M. Borkovec and G. Trefalt, Phys. Rev. Lett., 2016, 117, 088001.

51 A. D. Nikolov and D. T. Wasan, Langmuir, 1992, 8, 2985-2994. 